\title{
Early Stage Disease Diagnosis System Using Human Nail Image Processing
}

\author{
Trupti S. Indi \\ Assistant Professor, Department of Information Technology, Walchand Institute of Technology, \\ Solapur, Maharashtra, India \\ E-mail: tupshi2006@gmail.com
}

Yogesh A. Gunge

PG Student, Department of Computer Science \& Engineering, College of Engineering Pune, Pune, Maharashtra, India E-mail: gungeyogesh25@gmail.com

\begin{abstract}
Human's hand nail is analyzed to identify many diseases at early stage of diagnosis. Study of person hand nail color helps in identification of particular disease in healthcare domain. The proposed system guides in such scenario to take decision in disease diagnosis. The input to the proposed system is person nail image. The system will process an image of nail and extract features of nail which is used for disease diagnosis Human nail consist of various features, out of which proposed system uses nail color changes for disease diagnosis. Here, first training set data is prepared using Weka tool from nail images of patients of specific diseases. A feature extracted from input nail image is compared with the training data set to get result. In this experiment we found that using color feature of nail image average $65 \%$ results are correctly matched with training set data during three tests conducted.
\end{abstract}

Index Terms - Nail, color analysis, weka, classifier, image processing.

\section{INTRODUCTION}

In healthcare domain many diseases can be predicted by observing color of human nails. Doctors observe nails of patient to get assistance in disease identification. Usually pink nails indicate healthy human. The need of system to analyze nails for disease prediction is because human eye is having subjectivity about colors, having limitation in resolution and small a mount of color change in few pixels on nail would not be highlighted to human eyes which may lead to wrong result where as computer recognizes small color changes on nail [7].

The proposed system will extract color feature of human nail image for disease prediction. The system is focusing on image recognition on the basis of human nail color analysis. Many diseases could be identified by analyzing nails of human hands. In this system human nail image is captured using camera. Captured image is uploaded to our system and region of interest from nail area is selected from uploaded image manually. The selected area is then processed further for extracting features of nail such as color of nail. This color feature of nail is matched using simple matcher algorithm for disease prediction. In this way the system is useful in prediction of diseases in their initial stages.

In literature study we mentioned some of the diseases with its related color change in nails.

\section{BACKGROUND}

There are different ways available in healthcare domain to diagnose the diseases in human body. Analysis of human nail's color is one of the ways to predict or ensure the existence of disease. Human nails plays important role for predicting diseases with nail's color changes, shape changes etc. Pink nails indicate healthy human and a particular color changes in nails indicates certain diseases. There are many diseases which can be predicted by color of nails such as pale nails indicates Anemia, Congestive heart failure, Liver disease, Malnutrition. Human nails provide useful information about disorders or any nutritional imbalances depending upon their shape, texture and color.

\section{A. Parts of Nail}

The figure-1 explains the typical human nail structure and it parts.

Lunula: It is the moon shape observed at the base of the nail plate but not always.

Cuticle: Over the base of the nail plate the flat of thin tissue is called Cuticle.

Nail Root: the proximal end of the nail under a fold of skin.

Nail Plate: It is s mooth, curved and light pink in color. It is the visible part of the nail.

\section{B. Nail Color Implications}

Pink Nails: Pink color nail indicate healthy nails which in turn indicates good health symptoms.

White Nails: Lack of iron and poor circulation, in which the blood is not reaching the end of your fingers, are resulting into white nails. It indicates anemic conditions or malnutrition. 
Red-purple nails: An upset digestive system caused by over consumption of sugar, pharmaceutical drugs, fruits and juices results into red-purple nails.

White Spots: White spots in nails indicate high content of sugar and lack of zinc which is required in the digestion process.

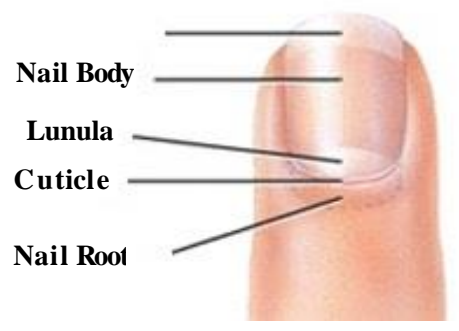

Fig.1. Parts of Nail

The proposed system is extracting color feature from nail image and stored in RGB form. For every disease case number of images taken as training set is between 10-15 samples per case. For classification Red, Green and Blue color average is calculated. Du ring matching phase, for input image RGB average is calculated and matched with training set data.

\section{RELATED WORK}

There are some existing systems which use color change of human palm for predicting disease. The system presented by Pandit et al. analyzes certain features in image and predicts probable disease using knowledge base of medical palmistry. Science of observing nails and palms to predict some diseases called Medical palmistry. Using example of the medical palmistry this model is discussed and presented for extraction of an interested portion of an image for further processing. This system presents work to get color of palm from the palm image and it works successfully on the different skin tones of human palm and increases accuracy of such observations of palm. The borders of palm are darker color than palm color in the scanned image of palm. Therefore exact boundary of palm may not found which can affect in the calculation of average color of palm. Image enhancement methods were used to overcome those problems. But this method is time consuming so to analyze nails it will take more time [3]. So our system is using digital camera or mobile camera to get good quality image therefore it is easy to extract features of nails.

There is another model, for nail color analysis which predicts diseases using digital image processing. The system presented by Hardik Pandit et. al observes the color of nails using the principles of medical science as basis and output of system is prediction of diseases, if found any. In healthcare domain doctors often observe human nails to as supporting information or symptoms in disease prediction. The same task is defined by the proposed model without any human intervention. The model gives more accurate results than human vision, because it overcomes the limitations of human eye like subjectivity and resolution power [7]

As mentioned earlier different colors of nails indicate certain diseases. To implement this model, authors used computer based reference color values of ill nails to compare user's nail. That is, color values of nails of user's input images would be compared with these reference colors. If the case matches with any of reference color values, user would be victim of that disease. There will be 50 samples per color are taken for reference color values of ill nails. For example, for yellow nails, 50 different yellow nails color values are considered, and then their arithmetic mean is considered as a reference color value for yellow nails to RGB color components of reference color for that disease [7].

Table 1. Diseases based on nail color and shape

\begin{tabular}{|c|c|c|c|}
\hline $\begin{array}{l}\text { Sr. } \\
\text { No. }\end{array}$ & Nail Type & Image & Possible Diseases \\
\hline i. & White Nails & & $\begin{array}{l}\text { i. Jaundice } \\
\text { ii. liver trouble } \\
\text { iii. Anemia. }\end{array}$ \\
\hline ii. & $\begin{array}{c}\text { Yellow } \\
\text { Nails [13] }\end{array}$ & & $\begin{array}{l}\text { i. lung disease } \\
\text { ii. diabetes or } \\
\text { psoriasis } \\
\text { iii. thyroid disease }\end{array}$ \\
\hline iii. & Bluish Nails & & $\begin{array}{l}\text { i. heart problems } \\
\text { ii. emphysema }\end{array}$ \\
\hline iv. & $\begin{array}{c}\text { Pale Nails } \\
\text { [13] }\end{array}$ & & $\begin{array}{l}\text { i. Anemia Congestive } \\
\text { heart failure } \\
\text { ii. Liver disease } \\
\text { iii. Malnutrition }\end{array}$ \\
\hline v. & $\begin{array}{c}\text { Dark Lines } \\
\text { Beneath the } \\
\text { Nail }\end{array}$ & & $\begin{array}{l}\text { i. } \\
\text { melanoma( dangerou } \\
\text { s type of ) skin cancer }\end{array}$ \\
\hline vi. & $\begin{array}{l}\text { Beau's } \\
\text { Lines }\end{array}$ & & i. systematic disease \\
\hline vii. & $\begin{array}{l}\text { Terry's } \\
\text { lines [15] }\end{array}$ & & $\begin{array}{l}\text { i. Hepatic failure } \\
\text { ii. Cirrhosis } \\
\text { iii. Diabetes } \\
\text { iv. Mellitus } \\
\text { v. Congestive Heart } \\
\text { failure } \\
\text { vi. Hyperthyroidism. }\end{array}$ \\
\hline
\end{tabular}

\section{PROPOSED SySTEM ARCHITECTURE}

There are different ways of disease diagnosis such as through various tests (blood test, urine test etc.) and symptoms available on various parts of body guides towards disease diagnosis. We are proposing a system which will take nail image as an input and output will be possible diseases prediction based on color changes. This is our first step towards a perfect system so we are not keen to accuracy of system.

The main objective of this system design is to provide an application for use in healthcare domain this is advantageous in terms of cost and time. The proposed 
system will take nail image as an input and will perform some processing on input image. Then finally it will predict probable diseases. This system can be used by people as well as by doctors in healthcare domain.

Table 2. Tests and Cost Required

\begin{tabular}{|c|c|c|}
\hline Sr. No. & Test Name & Cost (Rs) \\
\hline 1. & LFT(liver functioning test) & 600 \\
\hline 2. & $\begin{array}{c}\text { TFT(thyroid functioning test) T3 T4 } \\
\text { T SH }\end{array}$ & 500 \\
\hline 3. & BSLF+PP (BSL fasting BSL PP) & 100 \\
\hline 4. & KFT (creativity, urine) & 240 \\
\hline 5. & Urine(urine routine) & 60 \\
\hline 6. & $\begin{array}{c}\text { Lipid profile(cholesterol,triglycerides, } \\
\text { HDL) }\end{array}$ & 500 \\
\hline 7. & $\begin{array}{c}\text { CBC(WBC count, platelet count, HB } \\
\text { count) }\end{array}$ & 150 \\
\hline 8. & ESR(Erythrocyte Sediment Rate) & 60 \\
\hline
\end{tabular}

In healthcare domain to predict a disease, patient should go through various tests which are of high cost as mentioned in above table. The proposed system will help to avoid unwanted tests in the earlier stage of disease.

\section{A. Block Diagram of System}

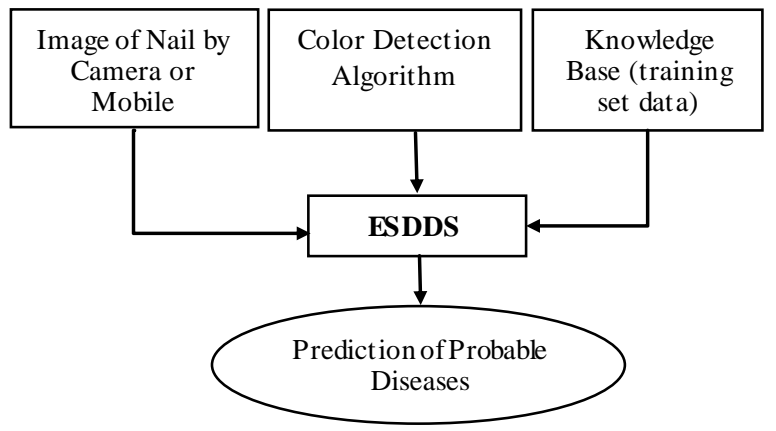

Fig.2. Block Diagram of ESDD System

Data Input Constraints:

i. Nails should be clean, having no any color like nail polish or any artificial marks on nails.

ii. Image should be taken under conditions like proper sunlight.

iii. Image background should be white or black background.

iv. Image should be taken without flash and clear.

\section{B. System Architecture}

The ESDDS systemwill consist of following steps:

i. Input nail image captured using digital camera (5 mega pixels) and uploaded to ESDD system.

ii. Through ESDD system's GUI select ROI of image for disease prediction.

iii. Send ROI image part and user in formation to server for processing.

iv. At server side, calculate average RGB color of ROI of nail image.

v. Extracting nail color in RGB format for input image.

vi. Server sends list of probable diseases which will be displayed at GUI in tabular format.

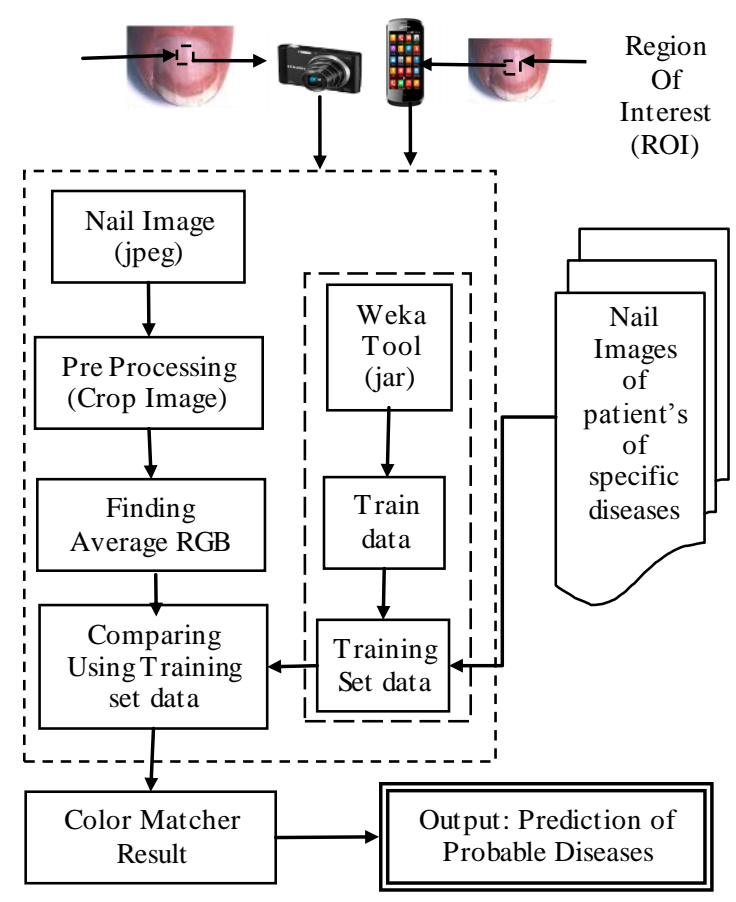

Fig.3. ESDD System Architecture

\section{Training Data Set Using Weka}

In ESDDS system, to train data (nail images of patients) proposed system using Weka tool [9] [10]. Here we have collected images of patients from hospitals. These images are given to imageUtils.java to find average RGB of that image and then it is given to diseaseclassifier.java to classify those average RGB values.

1. Input images taken from patients are trained using Weka tool.

2. In this we have used j48 classifier (c4.5 algorithm) to classify and train data available from patients.

3. An algorith $\mathrm{m} \mathrm{C4.5}$ is used to generate decision trees. The decision trees generated by $\mathrm{C} 4.5$ can be used for classification, and for this reason, C4.5 is often referred to as a statistical classifier.

Algorithm:

Step-I: Using a set of training data decision tree is build by $\mathrm{C} 4.5$ algorithm.

Step-II: C4.5 algorithm chooses the attribute of the data at each node of the tree that splits its set of samples into subsets impoverish in one class or the other.

Step-III: The criterion of splitting is the normalized information gain (difference in entropy).

Step-IV: The attribute with the highest normalized information gain is chosen to make the decision.

Step-V: C4.5 algorithm then recurs on the sub lists which are small in size. 
This algorithm has a few base cases.

Case-I: In the input list all the samples belong to the same class. In this case, it creates a leaf node for the decision tree saying to choose that class.

Case-II: None of the features provide any information gain. In this case, $\mathrm{C} 4.5$ creates a decision node higher up the tree using the expected value of the class.

Case-III: Instance of previously-unseen class encountered. Again, C4.5 creates a decision node higher up the tree using the expected value.

\section{RESULTS AND ANALYSIS}

\section{A. Graphical User Interfaces}

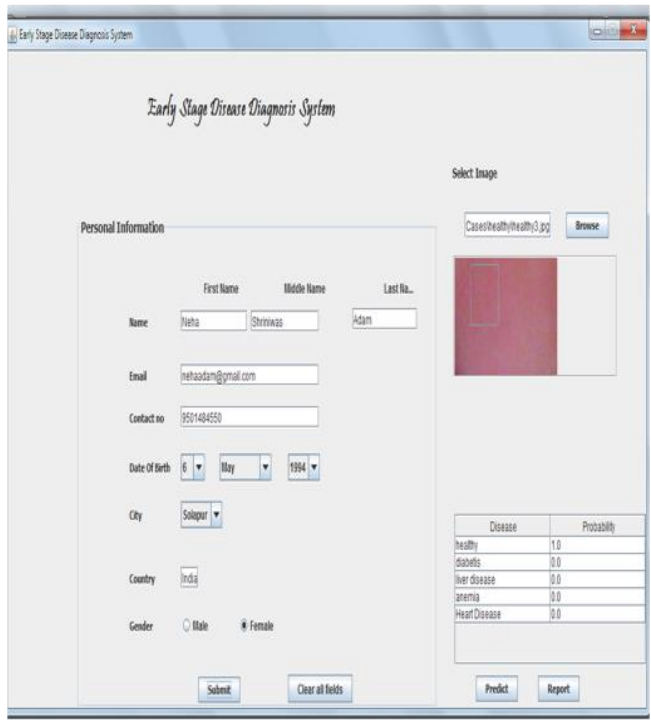

Fig.4. GUI for Desktop System
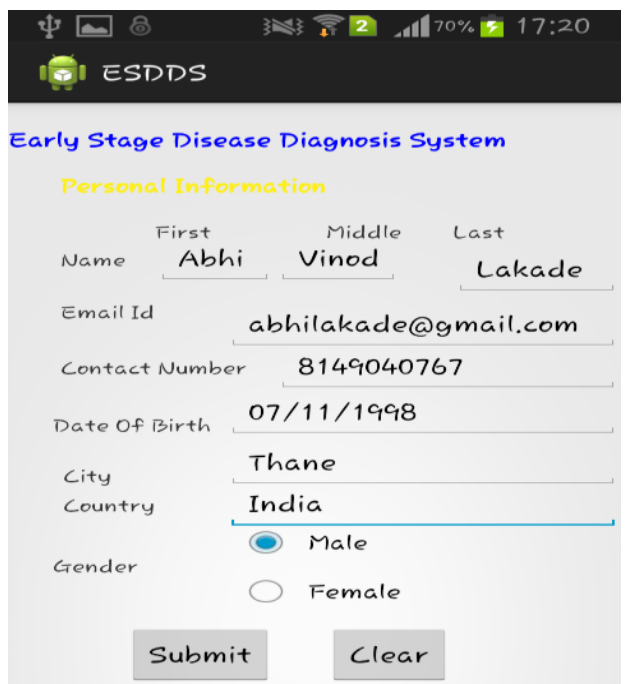

Fig.5. Snapshot of GUI of Android App

Fig-4 shows computer graphical user interface of presented system. Where nail images selected and cropped and submitted to the system along with patient details. On right bottom side of GUI the results are displayed.
Fig-5, 6, 7 and 8 shows the mobile graphical user interface of our ESDDS system.

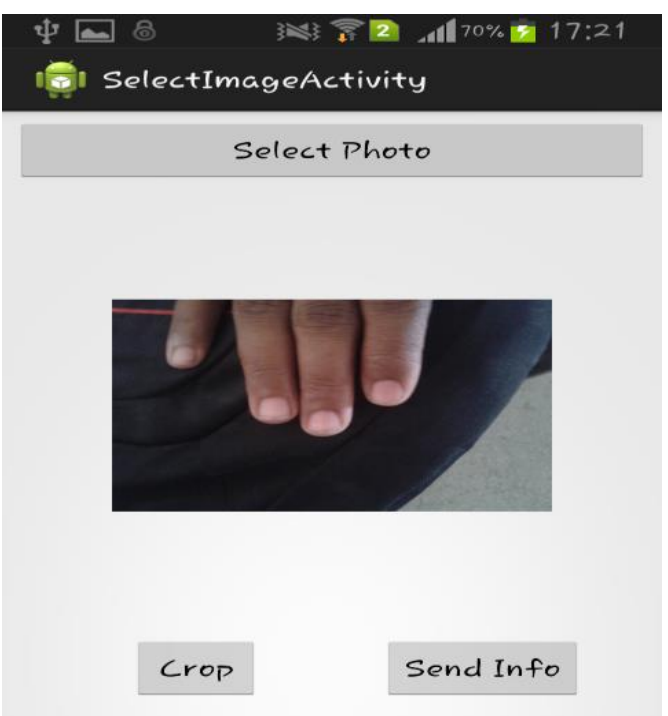

Fig.6. Android App - Image Capture

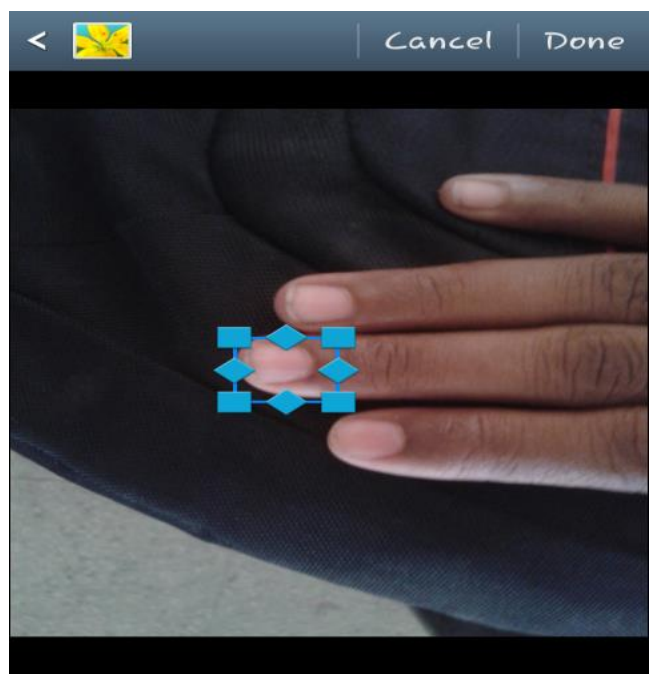

Fig.7. Android App - Cropping image
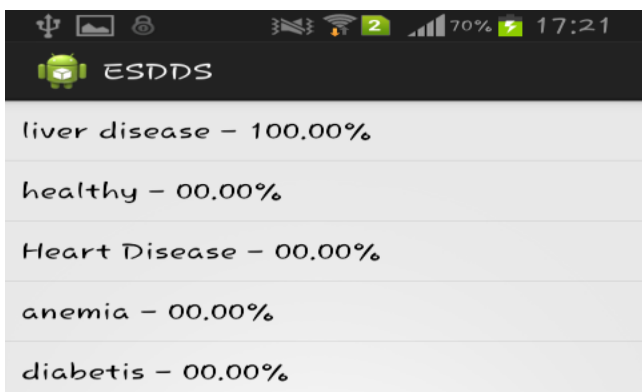

liver disease $-100.00 \%$

healthy $-00.00 \%$

Heart Disease - $00.00 \%$

anemia $-00.00 \%$

diabetis $-00.00 \%$ 


\section{B. Classifier Output}

Output of diseaseClassifier.java is shown in Figure 9.

Red $<=150$
$\mid$ blue $<=67$ : liver disease (8.0/1.0)
$\quad \mid$ blue $>67$
|| green $<=121$ : Heart Disease (16.0/1.0)
|| green $>121$
|| $\mid$ red $<=142$ : liver disease (8.0/1.0)
|| red $>$ 142: Heart Disease (2.0)
$\quad$ red $>150$
$\mid$ green $<=130:$ healthy (3.0)
$\mid$ green $>$ 130: liver disease (5.0)
Number of Leaves :
Size of the tree: 11

Fig.9. Classifier Output

\section{Results and Discussions}

Sample of input dataset and its respective average RBG values shown in Table 3.

Table 3. Sample Input Dataset and RGB values

\begin{tabular}{|c|c|c|}
\hline Sr. No & Image(heart disease) & RGB values \\
\hline 1. & & $150: 166: 155$ \\
\hline 2. & & $140: 134: 120$ \\
\hline 3. & & $127: 119: 111$ \\
\hline 4. & & $131: 101: 92$ \\
\hline 5. & & $129: 104: 99$ \\
\hline
\end{tabular}

\section{Test Cases Examined by ESDD System}

ESDD system tested with more than 100 nail image samples of 20 persons captured by digital camera as well as mobile camera in which 5 images per person taken. Some of nail images along with their results are shown in Table 4. Out of 100 samples, 20 samples of each disease - heart disease, diabetic disease, Liver disease, anemia disease and 20 samples of healthy person's nail image was considered.

The performance of ESDDS system is measured in terms of GAR and FAR. The genuine acceptance rate (GAR) is the ratio of truly matching samples and total number of tests. The false acceptance rate (FAR) is the ratio between the number of truly non-matching samples matched by the system and total number of tests [16]. Following Table 5 shows GAR and FAR rates generated for ESDDS system for diseases considered during this experiment:

Table 4. Sample Nail Images with Results

\begin{tabular}{|c|c|c|}
\hline Sr. No. & Image & Matching Results \\
\hline 1. & & Heart disease \\
\hline 2. & & Healthy nail 1.0 \\
\hline 3. & & Heart disease 1.0 \\
\hline 4. & & $\begin{array}{c}\text { Heart disease 0.9375,Liver } \\
\text { disease 0.0625 }\end{array}$ \\
\hline 5. & & Liver disease 1.0 \\
\hline
\end{tabular}

Table 5. Genuine Acceptance Rate (GAR) and False Acceptance Rate (FAR)

\begin{tabular}{|c|c|c|c|c|}
\hline $\begin{array}{c}\text { Sr. } \\
\text { No. }\end{array}$ & $\begin{array}{c}\text { Name of } \\
\text { Disease }\end{array}$ & GAR & FAR & $\begin{array}{c}\text { Average } \\
\text { Matched }\end{array}$ \\
\hline 1 & Anemia & 13.33 & 6.66 & $70 \%$ \\
\hline 2 & Diabetic & 12.66 & 6.66 & $65 \%$ \\
\hline 3 & Heart & 10.66 & 9.33 & $55 \%$ \\
\hline 4 & Liver & 11.33 & 9 & $50 \%$ \\
\hline 5 & Healthy & 16.33 & 3.66 & $85 \%$ \\
\hline \multicolumn{4}{|c|}{ Average } & $65 \%$ \\
\hline
\end{tabular}

\section{CONCLUSION AND FUTURE WORK}

\section{A. Conclusion}

There are five main parts of nail namely lunula, cuticle, nail root, nail plate, nail lines out of which we used nail plate is used in ESDD system. In presented system, system analyzes the human nail and gives probable disease for person including healthy case. Here, for disease prediction nail color (average RGB) value used as a nail feature. This model gives more accurate results than human vision, because it overcomes the limitations of human eye like subjectivity and resolution power.

\section{B. Future Work}

In presented work, nail color's RGB average value fro $m$ input image is used for classifying the diseases, but in future we can add some other feature of nail like pattern of nail for classifying the diseases. Along with these features we can also collect other symptoms observe in patient as an input to our system for disease prediction. Combining features of nails both color and pattern and other symptoms of patient's for more accurate results. It will be combination of textual features and features extracted from human body parts such as nail features etc. 


\section{REFERENCES}

[1] R. C. Gonzalez and R. E. Woods, "Digital Image Processing", 2nd edition, Pearson Education, 2004.

[2] Pandit Hardik and Shah D M: "A System for Nail Color Analysis in Healthcare", International Conference on Intelligent Systems and Signal Processing IEEE (ISSP2013), 1-2 March 2013, India (IEEE publisher).

[3] Pandit Hardik and Shah D M:"Application of Digital Image Processing and Analysis in Healthcare Based on Medical Palmistry", International Conference on Intelligent Systems and Data Processing (ICISD) 2011, Special Issue published by International Journal of Computer Applications (IJCA).

[4] Gurcharan Singh: "Nails in systemic disease", Raji's Skin Care Centre, Bangalore, India, Indian Journal of Dermatology, Venereology, and Leprology, NovemberDecember 2011 , Vol 77, Issue 6.

[5] Pandit Hardik and Shah D M: "The Model For Extracting A Portion Of A Given Image Using Color Processing", International Journal of Engineering Research \& Technology (IJERT), ISSN: 2278-0181, Vol. 1 Issue 10, December- 2012

[6] Dutta S. and Chaudhuri B.B.:"A Color Edge Detection Algorithm in RGB Color Space", Advances in Recent Technologies in Communication and Computing, 2009. ARTCom '09. International Conference on 2/7-28 Oct. 2009, Kottayam, Kerala (IEEE publisher).

[7] Pandit Hardik and Dipti Shah: "The Model of nail color analysis - An application of Digital Image Processing", International Journal of Advanced Research in Computer Science and Software Engineering (IJARCSSE), ISSN: $2277128 X$ Volume 3, Issue 5, May 2013.

[8] Sunanda Gupta, Charu Gupta and S.K. Chakarvarti "Image Edge Detection: A Review" International Journal of Advanced Research in Computer Engineering \& Technology (IJARCET) Volume 2, Issue 7, July 2013.

[9] Kumar Yugal and G. Sahoo: "Analysis of Parametric \& Non Parametric Classifiers for Classification Technique using WEKA", I.J. Information Technology and Computer Science, July 2012 MECS, 7, pp 43-49.

[10] Zdravko Markov Ingrid Russell: "An Introduction to the WEKA Data Mining System"

[11] http://mrishaanshareef.blogspot.in/2008/10/finger-nailsto-predict-health.html

[12] http://www.dailymail.co.uk/health/article-15289/Hownails-reveal-health.html

[13] Bhapkar P.H., Puttewar T.Y. and Patil R. Y.: "Topic Name - Nail Lacquers in Nail Diseases", IOSR Journal of Pharmacy, Volume 3, Issue 9 (October 2013), Pp 24-48, (e)-ISSN: 2250-3013, (p)-ISSN: 2319-4219.

[14] Fujishima Noriaki and Hoshino Kiyoshi: "Fingernail Detection System Using Differences of the Distribution of the Nail-Color Pixels", Journal of Advanced Computational Intelligence and Intelligent Informatics, Vol.17 No.5, 2013.

[15] Gurcharan Singh, Nayeem Sadath Haneef and Uday A: "Nail changes and disorders among the elderly", Indian Journal of Dermatology, Venereology and Leprology (IJDVL), Vol.71 No.6, pp 386-392, 2005.

[16] Indi T. S. and Raut S. D.: "Person identification based on multi-biometric characteristics", Emerging Trends in Computing, Communication and Nanotechnology (ICECCN), 2013 International Conference, pp 45-52, IEEE publisher.

\section{Authors' Profiles}

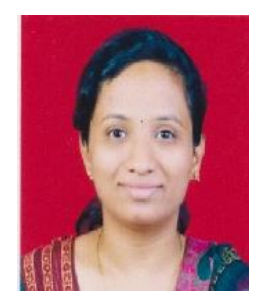

Indi Trupti S.: Assistant Professor at Walchand Institute of Technology Solapur University. Master of Engineering in Computer Science of Engineering, interested in major in image processing, data mining, databases.

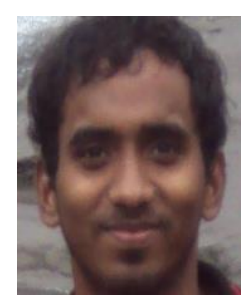

Gunge Yogesh A.: Bachelor of Engineering in Computer Science of Engineering from Pune University. Presently pursuing M. Tech., post-graduate degree in Computer Science of Engineering from Pune University, interested in image processing, machine learning and artificial intelligence, data mining.

How to cite this paper: Trupti S. Indi, Yogesh A. Gunge, "Early Stage Disease Diagnosis System Using Human Nail Image Processing", International Journal of Information Technology and Computer Science (IJITCS), Vol.8, No.7, pp.30-35, 2016. DOI: 10.5815/ijitcs.2016.07.05 